\title{
EDITORIAL
}

\section{TIPOLOGÍA DE ARTÍCULOS CIENTÍFICOS PUBLICABLES EN LA REVISTA Ipsa Scientia}

Para contribuir con el progreso de la ciencia, todo investigador debe trazarse como objetivo fundamental el dar a conocer los resultados obtenidos de sus investigaciones y compartirlos con la comunidad de pares, es por ello que participa en escenarios académicos y eventos científicos como conferencista o ponente en congresos, pero indudablemente se logra una mayor difusión con la elaboración de artículos científicos y su publicación en revistas arbitradas.

Normalmente, se sigue un esquema estandarizado para organizar el texto del artículo científico, compuesto por Introducción, Métodos, Resultados y Discusión (estructura IMRyD), seguido de unas conclusiones y las referencias bibliográficas, aunque cada revista establece sus propias normas de publicación y estilo, de acuerdo con su política editorial. En cuanto a la tipología de textos (Colciencias, 2010) que privilegian las revistas arbitradas colombianas, para fines de medición de indicadores por los organismos de acreditación, se encuentran los siguientes (Cardellach \& Ribera, 2007; Sánchez, 2011; Cuevas, 2013; Cevallos, 2015):

- Artículo de investigación: también denominado artículo original, representa un documento de un proyecto terminado de investigación empírica, que permite replicar el experimento o procedimiento, y sus resultados con el margen de error descrito, y repetir las observaciones, las deducciones teóricas y juzgar sus conclusiones.

- Artículos de reflexión: es un documento redactado a partir de los resultados de un proyecto de investigación terminada, a partir de una perspectiva crítica e interpretativa del autor, sobre un tema específico, recurriendo a fuentes originales.

- Artículo de revisión; también conocido como reseña, es un documento resultado de investigación completada, donde se profundiza en un tema muy especializado, revisando exhaustivamente el conocimiento disponible al respecto y construyendo un análisis sistematizado e integrado con argumentos fundamentados en la literatura científica existente, para abordar con claridad un problema actual.

En la revista Ipsa Scientia, además de los documentos antes mencionados, también se le da cabida al Artículo corto, un documento breve, que, aunque no califica para indexación, consideramos importante ya que presenta resultados preliminares o parciales de una investigación original que requieren de una pronta difusión. 


\section{EDITORIAL: Tipología de artículos científicos publicables en la revista Ipsa Scientia}

Iniciamos la presentación de nuestro tercer volumen de Ipsa Scientia con un artículo original que nos ofrecen los investigadores Fong, Colpas y Franco, titulado Academic performance and its association with class attendance, intrinsic motivation and gender in engineering students; cuyo objetivo fue analizar la asociación entre el rendimiento académico, asistencia a clases, motivación intrínseca y género en una muestra de 950 estudiantes de ingeniería de la Universidad de Cartagena, en el período 2014 - 2016. Los resultados obtenidos evidencian una significancia estadística importante entre el rendimiento académico y la motivación intrínseca, más no se pudo determinar su relación estadística con las variables asistencia a clases y género. En conclusión, los indicadores de motivación inciden de manera importante en el rendimiento que exhiben los estudiantes de ingeniería.

En segundo lugar, los investigadores Muñoz, Vargas y Latta, estudiaron la Estructura del clima organizacional en algunas universidades públicas del norte de Colombia, con el objetivo de identificar la estructura del clima organizacional presente en las universidades públicas del departamento de La Guajira. Aplicaron un cuestionario auto administrado, conformado por preguntas tipo escala Likert, cuyos resultados evidencian las falencias en las líneas de mando formales e informales, conllevando con esto el no cumplimiento de objetivos y metas comunes propuestas, donde la confluencia de las diferentes partes se convierta de ventajas competitivas sustentables.

Por otra parte, Jaimes, nos ofrece su artículo titulado Sistema de gestión de seguridad y salud en el trabajo: una revisión desde los planes de emergencia, donde realizaron una revisión sobre investigaciones recientes relacionadas con el sistema de gestión de seguridad y salud en el trabajo, principalmente en materia de planes de emergencia a partir del análisis de la literatura científica disponible. El estudio proporciona herramientas teóricas para las instituciones en general comprometidas con la implementación de la gestión de la seguridad y salud laboral, los autores plantean como un desafío presente la apropiación de políticas que genere una cultura de autocuidado integral en cualquier nivel de gestión.

Seguidamente, Palacio, Ahumada, Orjuela y Posada, en su artículo Towars a strategic epidemiology of occupational safety and health, realizaron una revisión documental sobre epidemiología estratégica para la seguridad y salud en el trabajo. Su objetivo fue analizar cualitativamente la literatura científica disponible en las bases de datos Scielo, Redalyc y páginas web oficiales, utilizando como palabras de búsqueda: epidemiología, seguridad industrial y salud ocupacional. Se obtuvo información relevante relacionada con el objetivo propuesto, que se presenta en 3 apartados: la epidemiología de la seguridad y la salud; conceptos, medidas y estudios en epidemiología y desafíos presentes y futuros.

Por su parte, Romero y Castro, nos entregan su artículo denominado Calidad de vida en pacientes obesos sometidos a sleeve gástrico laparoscópico, cuyo objetivo fue determinar la calidad de vida en una muestra de 60 pacientes obesos sometidos a este tratamiento durante el período comprendido desde enero de 2012 a diciembre 2015, en el E.S.E Hospital Universitario del Caribe, Cartagena. Se aplicaron tres cuestionarios, y el análisis de los resultados obtenidos permite determinar que el SGL es el tratamiento de elección más efectivo para obtener una mejor calidad de vida luego de que otras medidas terapéuticas hayan fallado.

IPSA SCIENTIA - Vol. $3 \mathrm{~N}^{\circ} 1$ - 2018 


\section{EDITORIAL: Tipología de artículos científicos publicables en la revista Ipsa Scientia}

Finalmente, Lugo, Cáceres, Coquel, Ramos, Puello y Ruiz, en su artículo titulado Knowledge in Access to the Definitive and Vascular Peripheral Aerial of Prehospital Care Students, evaluaron el conocimiento del acceso a la vía aérea definitiva y vascular periférica, a través de un cuestionario aplicado a 84 estudiantes activos del programa de Tecnología de Atención Prehospitalaria de la Corporación Universitaria Rafael Núñez, matriculados entre el segundo y sexto semestre, para el segundo período del año 2017. Los resultados indican bajo nivel de conocimientos básicos para realizar procedimientos invasivos en la vía vascular periférica, destacando la falta de protocolos, manejo y función de soluciones cristaloides, equipos de venoclisis y catéteres, así como de los protocolos para el manejo de la vía aérea, del equipo de intubación endotraqueal, las indicaciones y contraindicaciones para la intubación endotraqueal definitiva y los beneficios que genera en los pacientes.

De esta manera, los artículos científicos que convergen en esta nueva entrega de la revista Ipsa Scientia, ofrecen resultados que representan un aporte al conocimiento dentro del campo del saber abordado por cada uno de ellos.

\section{PhD. José Alexander Linares Morales}

Editor

\section{Referencias}

Cardellach, F. \& Ribera, J. (2007). Tipos de artículo. En K. Mabrouki \& F. Bosch (Coord.) Redacción científica en biomedicina: Lo que hay que saber. (pp. 15-23), Barcelona, España: Fundación Dr. Antonio Esteve.

Cevallos, G. (2015). Manual de redacción científica. El artículo científico. Málaga: España: Servicios Académicos Intercontinentales S.L.

Colciencias (2010). Documento Guía Servicio Permanente de Indexación de Revistas de Ciencia, Tecnología e Innovación. Bogotá, Colombia: Base Bibliográfica Nacional - BBN Publindex.

Cuevas, A. (2013). Guía básica para publicar artículos en revistas de investigación. Guanajuato, México: Universidad de Celaya, Centro de Investigación.

Sánchez, A. (2011). Manual de redacción académica e investigativa: Cómo escribir, evaluar y publicar artículos. Medellín, Colombia: Católica del Norte - Fundación Universitaria.

IPSA SCIENTIA - Vol. $3 \mathrm{~N}^{\circ} 1$ - 2018 